\title{
Capacity Response Sets for Security-Constrained Unit Commitment with Wind Uncertainty
}

\author{
Joshua D. Lyon ${ }^{\mathrm{a}}$, Muhong Zhang ${ }^{\mathrm{a}}$, Kory W. Hedman ${ }^{\mathrm{b}, *}$ \\ ${ }^{a}$ Department of Industrial Engineering, Arizona State University, Tempe, AZ 85287, USA \\ ${ }^{b}$ School of Electrical, Computer and Energy Engineering, Arizona State University, P.O. Box 875706, Tempe, AZ \\ 85287-5706
}

\begin{abstract}
Power systems face growing uncertainty from intermittent renewable resources. This uncertainty makes it harder to schedule generators efficiently because the economics depend on unknown outcomes. This research modifies security-constrained unit commitment to anticipate the cost of dispatching backup capacity if and when it is needed. The model uses capacity constraints to cover individual scenarios by defining scenario response sets, which are revised iteratively using a mixed-integer program. The approach provides a mathematical way to derive capacity requirements like those used today in CAISO and ISO-NE. Testing on the IEEE 73-bus test case demonstrates the capacity requirements are economical compared to other deterministic policies from the literature.

Keywords: Operating reserve, optimization, power system economics, reserve requirements, unit commitment
\end{abstract}

${ }^{*}$ Corresponding author. Telephone: 1+(480) 965-1276. Email: kory.hedman@asu.edu 


\section{Introduction}

The way generators are scheduled has a big influence on power system efficiency. Many scheduling decisions are made through energy markets that can exchange millions of dollars per day. The standard practice is to optimize around a single assumed system state. However, this can lead to suboptimal decisions because realized costs may differ from projections when the system is stochastic. This paper proposes a heuristic algorithm to help system operators achieve better economics when uncertainty is high, such as when wind constitutes a large portion energy. The heuristic is applied to the securityconstrained unit commitment problem (SCUC), which is central to day-ahead energy markets across the United States.

Renewable portfolio standards are requiring up to $33 \%$ of energy to come from renewable resources by the year 2020 [1]. These requirements are accelerating investments in wind turbines. There will inevitably be large forecast deviations because day-ahead wind forecast errors often exceed $20 \%$ [2]. Deeper wind penetrations will require more aggressive rebalancing and, consequently, operating costs are likely to become more volatile. This increases the motivation for capturing scenario costs within SCUC to identify solutions that are economical across a range of scenarios.

Stochastic models provide advanced ways to address wind uncertainty. Stochastic programming has been applied for commitment decisions to minimize expected cost across a range of scenarios [3, 4, 5, 6, 7, 8. Two-stage robust optimization has been applied in a similar way to protect against extreme scenarios [9, 10, 11]. Robust optimization is being investigated by a major software vendor (Alstom Grid) for changing commitments within the operating day [12], but there is no apparent evidence for application in day-ahead markets at this time. Stochastic programming and robust optimization require computational advancements before they become tractable for day-ahead markets, where there are many binary decision variables influencing the solution time.

Energy markets are run today using deterministic SCUC models that hedge against uncertainty using reserve and capacity requirements. For example, references [8] and [13] require reserve to exceed a prescribed percentage of the wind forecast and [14] and [15] require reserve to cover wind fluctuations with a certain probability. The market models in California and New England also require backup capacity to be held in strategic areas [16, 17]. This paper is the first to propose a mathematical approach for developing such capacity requirements. This problem is difficult because backup capacity may be:

- expensive to dispatch,

- unable to ramp fast enough, or

- undeliverable due to transmission constraints. 
This paper addresses the above issues by adapting the idea of response sets from [18. If operators require capacity commitments before the scenario is known, the response set is defined as all capacity that may count toward that requirement. Response set allows operators to preposition capacity that can respond to scenarios efficiently. The proposed algorithm provides a mathematical approach for determining response sets. It works by iterating between SCUC and an update routine where a mixed-integer program (MIP) identifies and disqualifies capacity that cannot contribute to inexpensive scenario response. The contribution of this paper is that response sets are applied to capacity requirements, address wind uncertainty, and are determined based on dispatching cost rather than solely reliability. This extends beyond conventional requirements that only target feasibility.

Like established capacity requirements in [16] and [17, which are used in practice today, the proposed heuristic does not guarantee optimality. However, testing on the IEEE 73-bus test case shows a $0.5 \%$ improvement to expected cost over competing reserve requirements from the literature. The algorithm's performance appears to lie somewhere between the prevailing deterministic and stochastic models, both in terms of solution quality and computational performance.

Testing also suggests that upfront commitment decisions become less important when operators can respond to different scenarios by turning "fast" generators on or off. Our findings are consistent with other studies showing that even simple deterministic models can work well under such circumstances [19, 20. The flexibility from fast generators is important, yet the inability to consider that flexibility limits the accuracy of many robust optimization models from the literature [21. A distinguishing feature of the proposed approach is that modeling fast generators poses little additional computational challenge. This feature makes capacity response sets applicable across a wider range of systems and conditions and allows for operational continuity (e.g., in systems moving towards a greater number of fast generators).

The paper outline is as follows. Section 2 formulates the SCUC problem. Section 3 describes the form of the proposed capacity requirements, which replace the direct modeling of scenarios from the stochastic formulation. Section 4 presents the algorithm to determine response sets. Section 5 reports numerical results. Finally, Section 6 concludes and summarizes potential for future work.

\section{Unit Commitment Under Uncertainty}

The unit commitment problem is a model to optimize what generators will be turned on or off during future time windows. A special version of unit commitment is SCUC, which includes constraints protecting against uncertainty. There are deterministic and stochastic approaches to the problem. Deterministic SCUC is standard in industry today and often includes reserve requirements or capacity constraints. The stochastic model is a more general and accurate approach that optimizes across several 
scenarios $s \in S$ simultaneously. We start by describing the stochastic unit commitment problem $(S U C)$ before defining deterministic SCUC as a special case of the more general problem. The general problem definition is

$$
\begin{gathered}
(S U C): \text { Minimize } f(\boldsymbol{x}) \\
\text { Subject to: }
\end{gathered}
$$

Network constraints:

$$
\begin{array}{lr}
i_{n t}^{s}=\sum_{g \in G(n)} p_{g t}^{s}+k_{n t}^{s}+b_{n t}^{s}-D_{n t}^{s}, & \forall n \in N, t \in T, s \in S \\
\sum_{n \in N} i_{n t}^{s}=0, & \forall t \in T, s \in S \\
-F_{l} \leq \sum_{n \in N} P T D F_{n l} i_{n t}^{s} \leq F_{l}, & \forall l \in L, t \in T, s \in S
\end{array}
$$

Generator capacity and ramping constraints:

$$
\begin{array}{ll}
0 \leq k_{n t}^{s} \leq K_{n t}^{s} & \forall n \in N, t \in T, s \in S \\
P_{g}^{\min } u_{g t}^{s} \leq p_{g t}^{s} \leq P_{g}^{\max } u_{g t}^{s}, & \forall g \in G, t \in T, s \in S \\
-R_{g} \leq p_{g t}^{s}-p_{g, t-1}^{s} \leq R_{g}, & \forall g \in G, t \in T, s \in S
\end{array}
$$

Commitment constraints for stage two:

$$
u_{g t}^{s}=u_{g t}^{0}, \quad \forall g \notin G^{\mathrm{fast}}, t \in T, s \in S
$$

Other constraints and variable domains:

$$
\begin{aligned}
& G(\boldsymbol{x}) \leq 0 \\
& u_{g t}^{s} \in\{0,1\}, b_{n t}^{s} \geq 0, \quad \forall g \in G, n \in N, t \in T, s \in S .
\end{aligned}
$$

The commitment decisions in $(S U C)$ are coupled with an optimal power flow that anticipates how generators will be dispatched for each scenario. The nomenclature section defines the terms for this model and all other models in this paper. The objective (1) minimizes a given cost function $f(\boldsymbol{x})$, where $\boldsymbol{x}$ is the vector of decision variables. Alternative objective functions are described in Section 2.1 . The only difference between scenarios is a limit on the available wind production in (5), where $k_{n t}^{s}$ is wind production and $K_{n t}^{s}$ is the available wind power at node $n$ during period $t$.

Network constraints model locational injections (2), power balance (3), and transmission capacity limits (4). The power flows are modeled using power transfer distribution factors, where PTDF is the sensitivity of flow across line $l$ to injections from node $n$ to the reference node. This type of linear approximation is common in power production scheduling [22]. If the network constraints are difficult to satisfy, then flow may be balanced by the dummy variable $b$ at a penalty to the objective 
Table 1: Unit commitment problem definitions. The SCUC model is used throughout this paper.

\begin{tabular}{ll} 
Model & Definition \\
\hline Unit Commitment & Model to schedule generator commitments. \\
Stochastic Unit Commitment $(S U C)$ & Version that directly considers scenario response. \\
$\begin{array}{l}\text { Security-Constrained Unit Commit- } \\
\text { ment (SCUC) }\end{array}$ & $\begin{array}{l}\text { Version that prepares for scenarios via security constraints } \\
\text { (e.g., capacity constraints). Deterministic SCUC is defined } \\
\text { as problem }(S U C) \text { with } S=\{0\} .\end{array}$ \\
\hline
\end{tabular}

function (this penalty is discussed further in Section 2.1]. The remaining constraints are represented by $G(\boldsymbol{x}) \leq 0$ in $(9)$; they often include minimum up and down times, storage, interface limits, and reserve requirements to cover uncertainties not captured by scenarios $s \in S$ (such as resource outages and quick wind fluctuations at finer time intervals than the model considers).

This model is formulated as a two-stage decision problem. The first stage positions generators for base conditions (designated here as $s=0$ ) before the scenario is known. These decisions influence the second stage through constraints (8), which specify that only fast generators $g \in G^{\text {fast }}$ may change commitment status for scenario response. Two-stage models, such as this, assume uncertainty is revealed at a single point in time. More precise models in the literature can include three or more stages [3, 4], but these problems can be much harder to solve. The intent of stochastic models is to anticipate operating cost well enough to make informed first-stage decisions.

The first stage determines what capacity to commit from slow generators. All other decisions can be deferred until after the scenario is revealed. The same two-stage structure is used by [5, 6, 7, 8, 10, and [1], although some of these references do not model fast generators (effectively assuming $G^{\text {fast }}=\varnothing$ ).

We define deterministic SCUC to be $(S U C)$ with $S=\{0\}$, which effectively removes all second-stage scenarios and makes the problem much smaller and easier to solve. A typical assumption is that wind availability in the first stage $K_{n t}^{0}$ equals the best point forecast available when the problem is solved (although other more sophisticated approaches have been investigated [23]). The downside is that optimal solutions to this simplified problem may perform poorly for one or more scenarios. To compensate for not modeling scenarios, deterministic formulations will often acquire reserves or backup capacity that can respond if needed. One such policy is the basis of this research. A summary of the different unit commitment definitions used throughout this paper is outlined in Table 1 .

\subsection{Objective Function}

Production costs are an important component of the SCUC objective. Marginal costs are frequently modeled as convex functions [24] and, for simplicity, linear costs are used here. Parameter $c_{g}$ is the 
cost per unit of energy produced by generator $g$. Fixed costs $c_{g}^{\mathrm{NL}}$ and $c_{g}^{\mathrm{SU}}$ (no-load and start-up) are incurred whenever the generator is committed or started. Load imbalances are also allowed at a high penalty $\nu$. Taking everything together, the total cost for a single scenario $s$ is

$$
c^{s}(\boldsymbol{x})=\sum_{g \in G} \sum_{t \in T}\left(c_{g} p_{g t}^{s}+c_{g}^{\mathrm{NL}} u_{g t}^{s}+c_{g}^{\mathrm{SU}} v_{g t}^{s}\right)+\nu \sum_{n \in N} \sum_{t \in T} b_{n t}^{s} .
$$

The parameter $\nu$ is a linearized penalty for violating node balance constraints. This would translate into physical load shedding if the imbalance propagated into real-time. For this reason, $\nu$ is bounded below the value of lost load, which is estimated to fall within a wide range of $\$ 2000-25,000 / \mathrm{MWh}$ for most outages exceeding one hour [25]. Traditional load shedding is not the only interpretation. With the evolution of the smart grid, more customers may voluntarily curtail themselves at a cost. Operators may also reduce the expected load shedding by repairing unreliable market solutions (these actions also tend to also have a cost [26]). Given the many ways to mitigate load imbalances, the use of $\nu$ in this paper is intended as a rough penalty for not satisfying all constraints in the modeled scenarios. The analysis in Section 5 will evaluate how sensitive results are to the parameter $\nu$.

Costs can be combined in various ways to create different objective functions, such as

$$
\begin{aligned}
f(\boldsymbol{x})^{\text {stochastic }} & =\sum_{s \in S} \pi^{s} c^{s}(\boldsymbol{x}) \\
f(\boldsymbol{x})^{\text {robust }} & =\max _{s \in S}\left\{c^{s}(\boldsymbol{x})\right\} \\
f(\boldsymbol{x})^{\text {base }} & =c^{0}(\boldsymbol{x}) .
\end{aligned}
$$

Equation 12 is a stochastic programming objective that weighs the cost of individual scenarios by probabilities $\pi$. Equation $(13)$ is a robust objective that measures the most expensive scenario in the uncertainty set. Equation (14) is a deterministic objective that only measures cost of first-stage decisions (the base case). Deterministic models neglect second-stage costs and may perform poorly across scenarios. However, their performance may be improved by committing backup capacity for scenario response. The proposed model uses objective (14) because only the base scenario $s=0$ is modeled directly in SCUC (using the definition in Table 1). The costs across other scenarios are addressed by proxy through capacity constraints derived on an iterative basis.

\section{Capacity Constraints: Practice and Proposal}

Operators do not apply the full stochastic problem $(S U C)$ to day-ahead markets today because it is too hard to solve in the alloted time. Instead, they protect against uncertainty using deterministic policies like reserve and capacity constraints. Capacity requirements were investigated as early as the 1960s by operators in PJM [27] and have since been integrated into day-ahead markets by operators in 
California (CAISO) and New England (ISO-NE) [16, 28. Their inclusion in day-ahead markets can lead to more efficient decisions. Capacity requirements can also improve the price convergence between day-ahead and real-time markets by better addressing reliability needs within the market model and reducing how many out-of-market adjustments are necessary to repair unreliable solutions [16, 17].

The capacity requirement used by CAISO is equivalent to

$$
\sum_{g \in \tilde{G}(j)} \mathcal{C}_{g t} u_{g t}^{0} \geq Q_{j t}, \quad \forall j \in J, t \in T
$$

where $\mathcal{C}_{g t}$ is the qualified capacity from generator $g$ and $Q_{j t}$ is the minimum amount of committed capacity required in area $j$ during period $t$ [16]. These parameters are tuned by the system operator to provide the desired amount of protection. Operators may apply capacity requirements for the whole system or to local areas $j \in J$, where $\tilde{G}(j)$ comprises generators in the respective area [16. The proposed model below adopts a similar policy:

$$
\sum_{g \in G} \mathcal{C}_{g t}^{s} u_{g t}^{0} \geq Q_{t}^{s}, \quad \forall s \in S, t \in T
$$

where

$$
Q_{t}^{s}=\sum_{n \in N}\left(D_{n t}^{s}-K_{n t}^{s}\right), \quad \forall s \in S, t \in T
$$

The primary difference from $(15)$ is that 160 is indexed by scenario $s$. Some capacity may be discounted $\left(\mathcal{C}_{g t}^{s}<P_{g}^{\max }\right)$ for one scenario but not another if dispatching constraints make the capacity expensive or unavailable. Constraint (17) sets the minimum quantity just high enough to cover demand minus wind production.

The response set for a scenario is defined by its qualified capacity $\mathcal{C}$. Figure 1 illustrates what one response set might look like in a hypothetical system. The map's lightly shaded regions contain capacity that is unavailable due to congestion. The bar chart identifies other reasons capacity may also be disqualified. The ramping and transmission criteria arise from physical constraints blocking the use of capacity in the respective scenario-period. The expense criterion arises when it is costly to utilize the capacity during a coordinated dispatch; note that even generators with low productions costs may be classified as expensive if they restrict production from other unit:11. A mathematical model for identifying and disqualifying unavailable or expensive capacity is proposed in Section 4

\footnotetext{
${ }^{1}$ For example, injections are generally less valuable at nodes with small locational marginal prices (LMPs) due to congestion. Generation is less valuable where LMPs are small because the LMP reflects the marginal value of supply at the given location. Generators with high productions costs may still be valuable if the LMP exceeds their marginal cost. The proposed algorithm in Section 4 classifies expensive capacity based on the overall cost of dispatching the entire system and not just the marginal cost of the respective generator.
} 

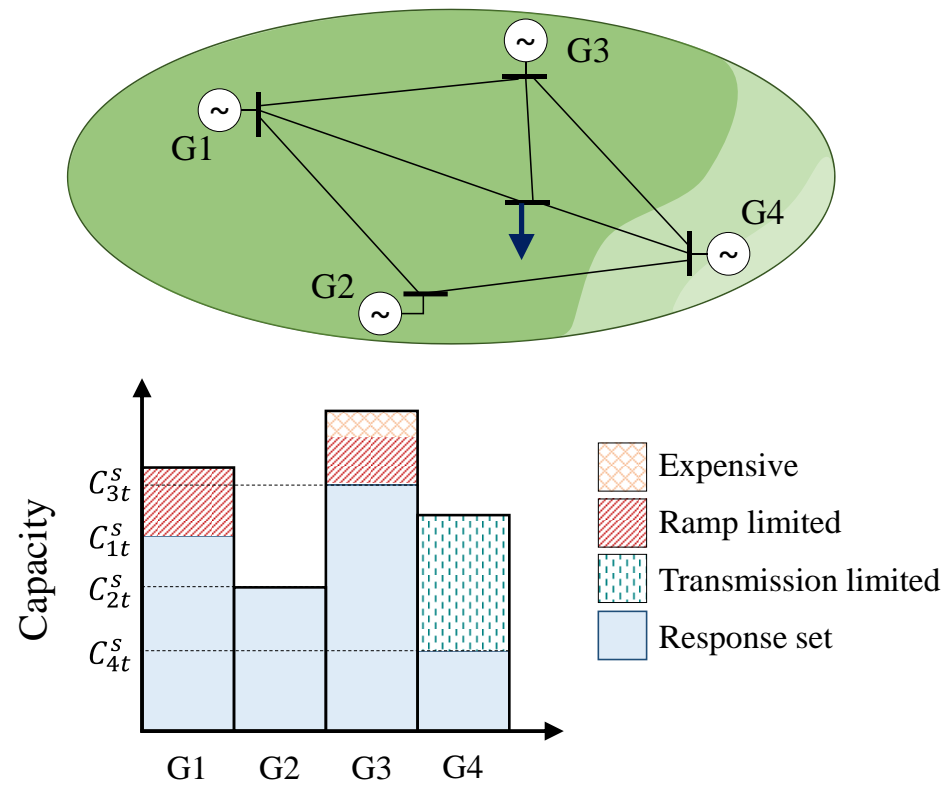

Figure 1: Stylized response set for a particular scenario-period $(s, t)$.

Constraint (18) below describes a more general requirement that accounts for uncommitted fast generators which may be turned on during scenario response:

$$
\sum_{g \in G} \mathcal{C}_{g t}^{s} u_{g t}^{0}+\sum_{g \in G^{\text {fast }}} \mathcal{U}_{g t}^{s}\left(1-u_{g t}^{0}\right) \geq Q_{t}^{s}, \quad \forall s \in S, t \in T,
$$

where the parameter $\mathcal{U}_{g t}^{s}$ is the capacity from uncommitted generator $g$ that is qualified for scenario $s$. The proposed algorithm determines both $\mathcal{C}$ and $\mathcal{U}$ to help ensure that efficient capacity is committed for scenario response. The heuristical nature of this approach arises from the entire recourse problem being reduced to a single constraint for each scenario-period. Nonetheless, the heuristic is of practical value because it is closely related to existing models used in industry today [16, 28. It also introduces a new formalism to capacity constraints by determining response sets in a mathematical way.

\section{Response Set Determination}

The response sets in (18) are similar to existing capacity constraints in CAISO and ISO-NE. The major difference is that they can change to meet the needs of individual scenarios. One scenario may create congestion into a local area and another may create a counterflow in the opposite direction. This section describes an algorithm for revising response sets to fit the needs of individual scenarios. The flowchart in Figure 2 describes the overall algorithm. Starting with initial response set definitions, the algorithm solves deterministic SCUC and evaluates the solution's performance across scenarios. If the 


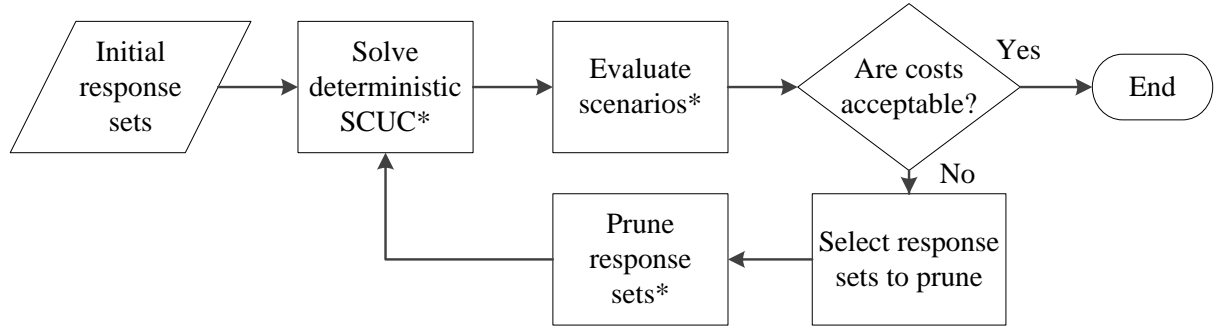

Figure 2: Decomposition algorithm for unit commitment with response sets (*requires an MIP solver).

base commitments do not work well for some scenarios, the response sets are pruned $(\mathcal{C}$ and $\mathcal{U}$ are made smaller) to remove capacity that was less helpful than expected. The revised response sets are reapplied through constraint $(18)$ in the next iteration to encourage SCUC to commit more preferable slow generators moving forward.

The process requires an MIP solver, such as Gurobi or CPLEX, to progress through the various steps. Individual steps from the flowchart in Figure 2 are now described in detail.

\subsection{Solving $S C U C$}

The first step of the algorithm is to solve deterministic SCUC with capacity requirements (16)-(18). The response sets must be initialized for the first iteration; an acceptable approach is to allow all capacity in every response set, although operators may choose to disqualify capacity upfront based on their knowledge of the system. The unit commitment decisions $u^{0}$ from SCUC will later be used as inputs to the following subroutines to evaluate the solution and determine what capacity to disqualify.

Solving SCUC is the computational bottleneck due to its size and the number of binary decision variables. The system operator MISO reports typical solution times to be within 3-15 minutes [29]. These computing times are important because SCUC is solved at each iteration of the proposed algorithm.

\subsection{Evaluating Scenarios and Stopping Criteria}

The evaluation subroutine assesses performance of the first-stage SCUC solution against different scenarios. This evaluation fixes the base commitments $u^{0}$ and solves the second stage of problem $(S U C)$. The computing times are not a big concern here because scenarios can be evaluated in parallel since $u^{0}$ is fixed in 8 based on the SCUC solution.

The algorithm terminates when costs from the evaluation subroutine are deemed acceptable. This paper adopts cost thresholds based on scenario regret, i.e., the cost above what could be obtained if a perfect forecast were available. The tolerable percent regret $\bar{R}$ is applied for each scenario relative to a 
lower bound $\underline{C}$ on the perfect-forecast solution:

$$
c^{s}(\boldsymbol{x}) \leq(1+\bar{R}) \underline{C}^{s}, \quad \forall s \in S .
$$

Scenarios that do not satisfy the cost threshold are placed in set $\tilde{S}$ to be pruned (by definition, $\tilde{S} \subseteq S$ will only contain scenarios with unsatisfactory costs in the current iteration). Constraining regret is a heuristic for reducing expected regret, which is equivalent to reducing expected cost. This approach is generally more risk averse than stochastic programming and more risk neutral than robust optimization 30. Regret is estimated here relative to a lower bound on the perfect-forecast solution $\underline{C}^{s}$ as this bound can be calculated efficiently by most MIP solvers.

There is no guarantee the above stopping criterion will be achievable for arbitrary $\bar{R}$. Optimistic targets could lead the algorithm to continually disqualify capacity in vain. It is advised that an additional stopping condition be adopted that terminates the algorithm when response sets shrink below a reasonable size. The situation will be apparent if the problem becomes infeasible or if objective values inflate due to constraint 18 becoming unreasonably strict. In order to avoid these situations, some prudence is necessary to select an $\bar{R}$ that captures opportunities without being overly ambitious.

\subsection{Pruning Response Sets}

At this point, the algorithm has identified expensive scenarios and placed them in set $\tilde{S}$ to be pruned. The qualified capacity coming from committed generators and uncommitted fast generators is now represented as

$$
\hat{P}_{g t}^{s}= \begin{cases}\mathcal{C}_{g t}^{s}, & \text { if } u_{g t}^{0}=1 \\ \mathcal{U}_{g t}^{s}, & \text { if } u_{g t}^{0}=0 \text { and } g \in G^{\text {fast }} \\ 0, & \text { if } u_{g t}^{0}=0 \text { and } g \notin G^{\text {fast }}\end{cases}
$$

The pruning subroutine identifies the portion of this response set capacity $\hat{P}_{g t}^{s}$ that cannot be dispatched at low cost given the incumbent base commitments $u^{0}$. The mathematical model for identification of costly capacity $(I C C)$ is

$$
(I C C) \text { : Minimize } \sum_{g \in G} \sum_{t \in T} \sum_{s \in \tilde{S}} \hat{P}_{g t}^{s} \gamma_{g t}^{s}
$$

subject to: $(3)-10]$

$$
\begin{array}{lr}
c^{s}(\boldsymbol{x}) \leq(1+\bar{R}) \underline{C}^{s}, & \forall s \in \tilde{S} . \\
i_{n t}^{s}=\sum_{g \in G(n)} p_{g t}^{s}+\sum_{g \in G} m_{g n t}^{s}+k_{n t}^{s}+b_{n t}^{s}-D_{n t}^{s} & \\
& \forall n \in N, t \in T, s \in \tilde{S}
\end{array}
$$




$$
\begin{array}{lr}
\sum_{n \in N} m_{g n t}^{s} \leq \hat{P}_{g t}^{s}-p_{g t}^{s}, & \forall g \in G, t \in T, s \in \tilde{S} \\
\sum_{n \in N} m_{g n t}^{s} \leq \hat{P}_{g t}^{s} \gamma_{g t}^{s}, & \forall g \in G, t \in T, s \in \tilde{S} \\
\sum_{g \in G} m_{g n t}^{s} \leq \sum_{g \in G(n)} \mathcal{C}_{g t}^{s}\left(1-u_{g t}^{0}\right), & \forall n \in N, t \in T, s \in \tilde{S} \\
m_{g n t}^{s} \geq 0 . & \forall g \in G, n \in N, t \in T, s \in \tilde{S}
\end{array}
$$

A solution to $(I C C)$ can be found using an MIP solver. The problem resembles the second stage evaluation model with three differences. First, the scenario costs are constrained to be below the maximum regret 22 . Second, generator capacity is limited to what currently belongs in the response set 24. Finally, costly capacity may be replaced by dummy injections: the variable $m_{\text {gnt }}^{s}$ replaces capacity from generator $g$ with an injection at node $n$. This mechanism is key to how the model works because the dummy injections identify capacity in the response set that cannot be dispatched without violating the cost constraint 22 . The proportion of a generator's capacity replaced by dummy injections is tracked by $\gamma$ through 25), and the objective function (21) minimizes this value so dummy injections are only used as a last resort to satisfy the cost and dispatching constraints. In this way the model identifies what response set capacity cannot contribute to an efficient scenario response given the first-stage decisions. The idea of using dummy injections is adapted from [18]: here they can be interpreted capacity from slow generators that may be committed in future iterations when a stricter capacity requirement will be used. As such, 26 limits dummy injections to locations that currently have uncommitted capacity from slow generators.

Let $\gamma^{*}$ be the optimal solution of problem $(I C C)$. This value represents the proportion of capacity that could not be dispatched while satisfying the cost constraint. The response sets are then pruned based on $\gamma^{*}$ according to

$$
\begin{aligned}
& \mathcal{C}_{g t}^{s(\text { new })}= \begin{cases}\mathcal{C}_{g t}^{s}\left(1-\gamma_{g t}^{s^{*}}\right), & \text { if } u_{g t}^{0}=1 \\
\mathcal{C}_{g t}^{s}, & \text { if } u_{g t}^{0}=0,\end{cases} \\
& \mathcal{U}_{g t}^{s(\text { new })}= \begin{cases}\mathcal{U}_{g t}^{s}\left(1-\gamma_{g t}^{s^{*}}\right), & \text { if } u_{g t}^{0}=0 \\
\min \left\{\mathcal{U}_{g t}^{s}, \mathcal{C}_{g t}^{s(\text { new })}\right\}, & \text { if } u_{g t}^{0}=1 .\end{cases}
\end{aligned}
$$

The capacity of each response set member is discounted in 28) and 29) by the proportion replaced by dummy injections in problem $(I C C)$. The same capacity cannot count toward capacity requirements in future iterations. A slightly modified rule is used in 29) for uncommitted fast generators to enforce $\mathcal{U} \leq \mathcal{C}$. This relation speeds up convergence and is justified because fast generators are more expensive when they have not been committed due to their fixed start-up costs. 
The computing needed to prune response sets is mild. Problem $(I C C)$ is trivially parallelizable because the scenarios are independent. The model is comparable to the evaluation sub-routine except for the inclusion of dummy injections $m$. Most of these additional variables must equal zero since (26) forbids dummy injections where there is no qualified capacity. For this reason, problem $(I C C)$ has similar computational requirements as the evaluation sub-routine. Both problems are relatively easy to solve quickly because scenarios are independent and because there have limited binary commitment decisions in the second-stage.

\section{Analysis and Results: IEEE 73-Bus Test Case}

Testing is performed on a 73-bus test case released by the Institute of Electrical and Electronics Engineers (IEEE). The test case is described by [31] and is available from 32. It is common to modify IEEE test cases. The analysis in this paper follows [33] by removing line (11-13), shifting $480 \mathrm{MW}$ of load from buses 14, 15, 19, and 20 to bus 13, and decreasing the capacity of line (14-16) to 350 MW. These modifications are made to each of the three identical areas that comprise the system. The removal of lines and shifting of load was originally done by [34] and the changing of line capacities by [35. This test case does not have strong congestion patterns, so a small amount of congestion is induced as in [36] by tripling the capacity of inexpensive hydro power in the area consisting of buses 1-24 and removing hydro from all other areas.

Wind farms are placed at nodes 23 and 47, which are central locations containing $660 \mathrm{MW}$ of coal generation each. The wind is placed at central parts of the system because isolated areas would more likely require additional transmission investments that are beyond the scope of this paper. Wind scenarios are generated as in [37] using data sampled from [38. The developed wind scenarios generate power up to $3340 \mathrm{MW}$ and energy equal to $33 \%$ of the average load.

Each test instance comprises 24 hours. The SCUC formulation includes minimum up and down times constraints as in 39. To control flexibility near the boundaries of the time horizon, conditions at the end of the day are treated as starting conditions for the beginning of the same day. Contingency reserves are enforced using the two reserve zone requirement from [18] with reserve sharing parameter $\alpha=0.5$. Load imbalances are allowed with $\nu=\$ 10,000 / \mathrm{MWh}$ (see Section 2.1 for interpretations of this parameter).

Testing is performed using the optimization software CPLEX v12.5 on a machine with an 8-core $3.6 \mathrm{GHz}$ processor and $48 \mathrm{~GB}$ of memory. The SCUC problem is terminated after five minutes or upon reaching an optimality gap of $0.1 \%$. Regret is calculated in each case relative to a tight lower bound $\underline{C}^{s}$ on the perfect-forecast solution. 


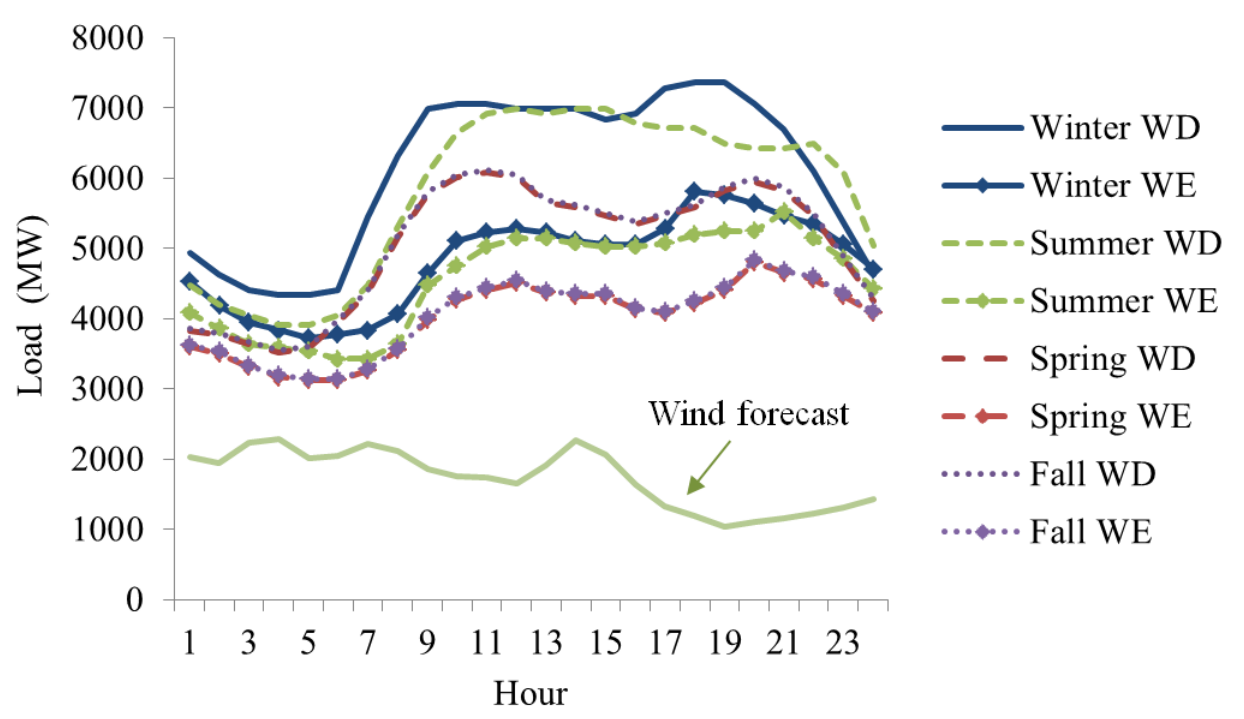

Figure 3: Load profile for the eight test days.

\subsection{Analysis Design}

\subsubsection{Test Days}

This analysis examines a portfolio of days including a weekday (WD) and weekend (WE) from each season. The load profile for each day is shown in Figure 3 . The same wind scenarios are used for each case, ranging in forecast from $28 \%$ of energy in Winter to $43 \%$ in Fall. The available wind energy is averaged across scenarios for the forecast $K_{n t}^{0}$ assumed in SCUC.

\subsubsection{Baseline Policies}

Several heuristics have been proposed in the literature to deal with wind uncertainty [40]. Many focus on mitigating short-term fluctuations ( $<20$ minutes). Others require reserve to exceed a percentage of the load or wind forecast at the hourly level [8, 13. A similar baseline policy is used here by coupling SCUC with the following constraints:

$$
\begin{array}{lr}
\sum_{g \in G} r_{g t}^{\text {slow }} \geq W \sum_{n \in N} K_{n t}^{0}, & \forall t \in T \\
p_{g t}^{0}-p_{g, t-1}^{0}+r_{g, t-1}^{\text {slow }} \leq R_{g}, & \forall g \in G, t \in T \\
p_{g t}^{0}+r_{g t}^{\text {slow }} \leq P_{g}^{\max }, & \forall g \in G, t \in T .
\end{array}
$$

The variable $r_{g t}^{\text {slow }}$ represents slow reserve from generator $g$ that is available within one scheduling period (60 minutes). Constraint (30) requires slow reserve to exceed a prescribed percentage $W$ of the wind forecast. Constraint (31) accounts for each generator's scheduled ramping [13]. Several values of $W$ are tested in this section and then analyzed post-hoc for which had the best performance. 
The baseline requirements are not enforced at a zonal level because the wind farms are centrally located in parts of the system that are generally export-constrained, making it easier to import reserves during wind shortages. The prime wind locations notwithstanding, local transmission constraints can still hinder reserve deliverability. A strong point of the proposed methodology is that it adapts to different congestion patterns across scenarios without relying on a single zone definition to work well under diverse conditions.

\subsubsection{Scenario Selection}

One challenge of the proposed algorithm is selecting a proper set of scenarios to model. Two contrasting approaches are used here. The first approach generates 50 random scenarios and the second approach targets extreme scenarios using scenario selection.

Various scenario selection techniques are proposed in the literature. Some identify scenarios that are representative of a larger set, e.g., [41, and others focus on capturing extreme scenarios, e.g., [6]. The latter approach is used here to hedge against costly load imbalances. A small training set is chosen to include:

1. the scenario with the smallest available wind energy,

2. the scenario with the smallest available wind energy during any single period,

3. the scenario with the biggest variance between periods,

4. the scenario with the biggest morning ramp,

5. the scenario with the biggest evening ramp,

6. the scenario with the biggest change in one period,

7. the scenario with the biggest difference between the maximum and minimum wind availability.

Seven scenarios meeting the above criteria are selected from 1000 randomly generated scenarios. Applying response sets for these scenarios helps prepare for extreme wind outcomes.

\subsubsection{Initial Response Sets}

The response sets are initialized with $\mathcal{C}_{g t}^{s}=\mathcal{U}_{g t}^{s}=P_{g}^{\max } \times(1-0.05)$ for all scenarios. This is chosen as a naïve starting point to demonstrate the capabilities of the algorithm. The $5 \%$ margin applied at the beginning is a subjective way to ensure there remains some capacity margin - this is based on the observation that capacity frequently exceeds load by at least a small amount in feasible solutions.

\subsubsection{Evaluating Solutions}

This analysis produces 64 SCUC solutions between the various baseline and response set policies. Given the first-stage SCUC commitments, each solution is evaluated by simulating dispatch across 100 random sample scenarios that are independent of the training scenarios. The evaluation solves 
Table 2: Results averaged across scenarios baseline policies ( $W \%$ of the wind forecast). The regret assumes $\nu=$ $\$ 10,000 / \mathrm{MWh}$.

\begin{tabular}{lrrrrrrrrrr}
\hline & \multicolumn{3}{c}{ Load Imb. (MWh) } & & \multicolumn{4}{c}{ Regret (\%) } \\
\cline { 2 - 3 } Day & $25 \%$ & $30 \%$ & $35 \%$ & $40 \%$ & & $25 \%$ & $30 \%$ & $35 \%$ & $40 \%$ \\
\hline Winter WD & 0.2 & 0.0 & 0.0 & 0.0 & 1.9 & 1.8 & 2.2 & 2.6 \\
Winter WE & 7.5 & 0.0 & 0.0 & 0.0 & & 15.9 & 2.0 & 2.2 & 2.4 \\
Summer WD & 0.8 & 0.0 & 0.0 & 0.0 & 3.0 & 2.2 & 3.0 & 3.3 \\
Summer WE & 0.1 & 0.1 & 0.1 & 0.0 & 2.7 & 2.7 & 2.9 & 3.2 \\
Spring WD & 0.1 & 0.0 & 0.0 & 0.0 & 2.4 & 2.4 & 2.9 & 3.5 \\
Spring WE & 14.7 & 6.6 & 0.0 & 0.0 & & 49.7 & 25.0 & 4.4 & 4.9 \\
Fall WD & 0.0 & 0.0 & 0.0 & 0.0 & 1.9 & 2.4 & 2.8 & 3.3 \\
Fall WE & 20.2 & 7.4 & 0.0 & 0.0 & 65.5 & 26.8 & 4.4 & 4.5 \\
\hline Average & 5.4 & 1.8 & 0.0 & 0.0 & 11.0 & 5.2 & 2.9 & 3.2 \\
\hline
\end{tabular}

a single 24-hour model assuming the sample scenario is revealed upfront. The corresponding results are optimistic because, in reality, forecast errors still occur during the operating day. However, this approach is common in the literature [5, 6, 7, 8, 10, 11] and all solutions are compared in a consistent way.

\subsection{Results - No Fast Generators}

The IEEE 73-bus test case does not specify which generators can be classified as fast. Our analysis first studies the system with no fast generators. The baseline results are shown in Table 2 for several values of $W$. A trade-off appears where acquiring more reserve protects against load imbalance at a cost. The policy that is observed to minimize the expected cost is $W=35 \%$ of the wind forecast. This policy is the cheapest overall but can be far from the cheapest on individual days, suggesting there could be room for improvement by adapting a dynamic policy.

The same baseline policy of $W=35 \%$ minimizes the expected cost for several violation penalties $\nu$, as shown in Figure 4. The more optimistic policies only perform better when $\nu<\$ 2500 / \mathrm{MWh}$. Such small values may be characteristic of systems that have many flexible loads willing to reduce their consumption at a relatively low price.

The proposed capacity constraints are applied with a maximum regret of $\bar{R}=10 \%$ together with the slow reserve requirement 30 with $W=20 \%$. Table 3 summarizes the results after running the algorithm. Every day experiences load imbalances in the first iteration that are eventually eliminated. However, the performance is more modest when evaluated outside of the training set of scenarios. The use of scenario selection tends to improve regret by reducing load imbalances in the evaluation scenarios. Scenario selection reduces regret from $3.1 \%$ to $2.5 \%$. This is lower than the best baseline 


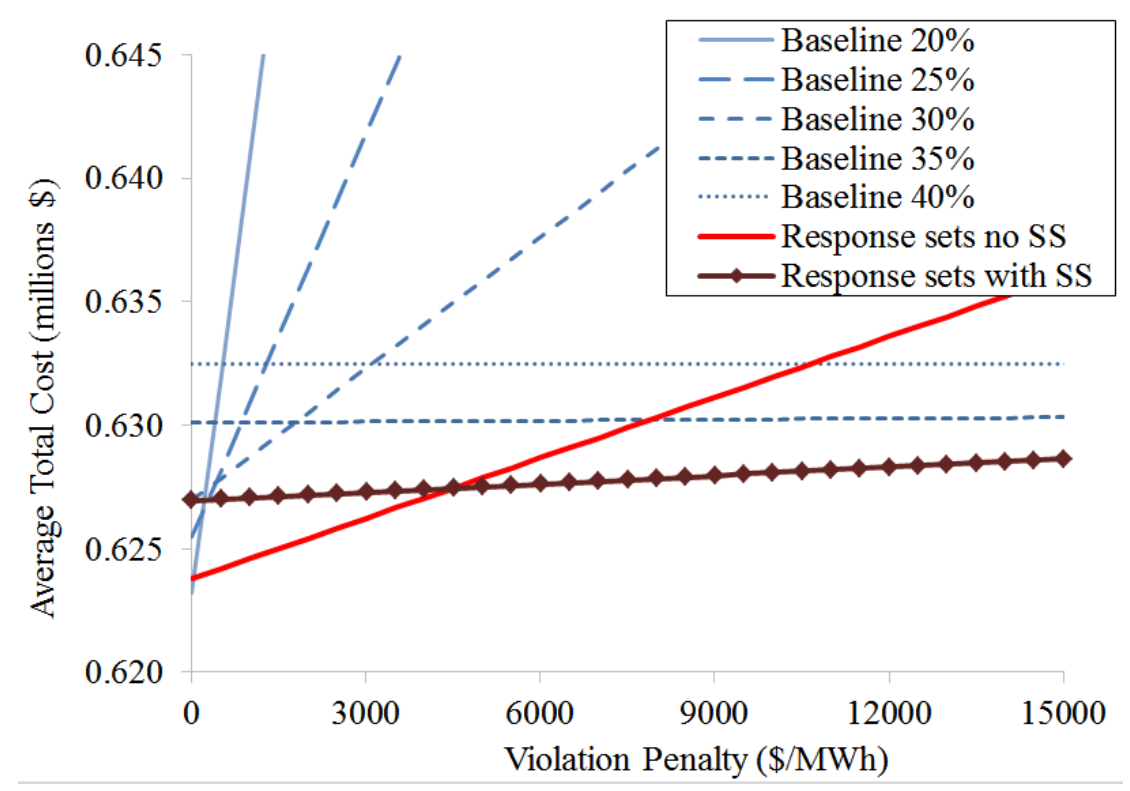

Figure 4: Average costs extrapolated for different violation penalties $\nu$. SS $=$ scenario selection.

policy. Furthermore, the expected cost is within $2.5 \%$ of the best possible stochastic solution since, by definition, no solution can have negative regret.

The performance is extrapolated for different violation penalties $\nu$ in Figure 4 The cost improvement over the baseline policies ranges from $0.2 \%$ to $0.5 \%$. The crossover point where the best baseline policy has a lower cost is $\nu=\$ 35,000 / \mathrm{MWh}$, which is higher than most estimates for value of lost load [25]. Systems with that high of a penalty on load imbalance may specify an uncertainty set with more extreme scenarios to further hedge against load imbalances.

A statistical analysis is applied to compare response sets with scenario selection to the baseline policy with $W=35 \%$. The average cost difference is approximately normally distributed and is analyzed here using the t-distribution 42 due to the statistically small sample size of eight days. The $95 \%$ confidence interval for the daily cost improvement is $\$(640,4671)$ for $\nu=\$ 5000 / \mathrm{MWh}$. The results indicate that the proposed policy may provide some cost benefit over the long run compared to simply requiring reserve to cover a percentage of the wind forecast.

\subsubsection{Computation}

The computational bottleneck in the proposed algorithm is the need to solve SCUC once every iteration. The evaluation and pruning stages solve relatively fast. The wall clock computing times are summarized in Table 4 A theoretical time for parallel computing is also computed by replacing the total evaluation and pruning times with the slowest scenario from each stage. The potential time reduction from parallel computing is modest (around 10\%) because most of the time is spent solving 
Table 3: Results averaged across scenarios for response set policies. The regret assumes $\nu=\$ 10,000 / \mathrm{MWh}$. SS $=$ scenario selection.

\begin{tabular}{lccccc}
\hline & \multicolumn{2}{c}{ Load Imb. (MWh) } & & \multicolumn{2}{c}{ Regret (\%) } \\
\cline { 2 - 3 } \cline { 5 - 6 } Day & No SS & With SS & & No SS & With SS \\
\hline Winter WD & 0.1 & 0.1 & & 1.5 & 1.9 \\
Winter WE & 0.0 & 0.0 & & 1.5 & 2.0 \\
Summer WD & 1.3 & 0.0 & & 3.2 & 2.2 \\
Summer WE & 0.1 & 0.0 & & 2.4 & 2.5 \\
Spring WD & 1.4 & 0.0 & & 4.1 & 2.3 \\
Spring WE & 1.2 & 0.3 & & 6.3 & 4.6 \\
Fall WD & 1.8 & 0.0 & & 4.4 & 2.2 \\
Fall WE & 0.7 & 0.6 & & 5.1 & 5.5 \\
\hline Average & 0.8 & 0.1 & 3.1 & 2.5 \\
\hline
\end{tabular}

SCUC. However, parallel computing would allow the model to consider many more scenarios without greatly increasing computation times.

The number of iterations are also shown in Table 4. One iteration of the algorithm has roughly the same solution time as the baseline policies. The proposed algorithm converges in as little as two iterations and as many as twenty-eight. This is slower than the baseline models but much faster than solving problem $(S U C)$ directly. It is also faster than results reported in the literature for fast stochastic programming solution methods like 8. An approximate comparison comes from iteration counts because [8] also solves SCUC at each iteration (it actually solves SCUC several times in parallel). The authors of 8 terminate their algorithm after 120 iterations, which is four times longer than the proposed algorithm. Some additional iterations can be attributed to [8] using a larger test case (their 225-bus model of the Western Interconnection has roughly 30\% more generators and three times more nodes), but the difference in iteration count is arguably large. This is just one example coming from a highly cited paper. The broad computational challenges of stochastic programming are well noted in the literature 43, 44] and are a big reason why capacity constraints are used in practice today.

Twenty-eight iterations is too many for application in day-ahead markets. The response sets should, therefore, be determined ahead of time or updated after the fact only to repair unreliable or inadequate solutions. Repairing unreliable solutions would be a natural first application while the computational performance is being improved. The above analysis does not explicitly evaluate this application but demonstrates the effectiveness of the proposed algorithm. The algorithm may be particularly well-suited to inform the manual commitments made by operators on a regular basis in ISO-NE [28. An automated approach such as this may locate capacity better and avoid the potential perception of biased decisions from market operators. 
Table 4: Iterations and computing time (theoretical times are shown in parenthesis for parallel computing). SS $=$ scenario selection.

\begin{tabular}{lcccccc}
\hline & \multicolumn{2}{c}{ Iteration Count } & & \multicolumn{2}{c}{ Time (min) } \\
\cline { 2 - 3 } \cline { 5 - 6 } Day & No SS & With SS & & No SS & With SS \\
\hline Winter WD & 10 & 10 & & $50(46)$ & $47(45)$ \\
Winter WE & 3 & 6 & & $7(5)$ & $17(16)$ \\
Summer WD & 11 & 13 & & $12(10)$ & $21(20)$ \\
Summer WE & 2 & 21 & & $14(13)$ & $35(32)$ \\
Spring WD & 11 & 31 & & $91(84)$ & $37(34)$ \\
Spring WE & 10 & 6 & & $39(36)$ & $37(36)$ \\
Fall WD & 5 & 28 & & $40(36)$ & $122(117)$ \\
Fall WE & 20 & 27 & & $115(105)$ & $140(135)$ \\
\hline Average & 9.0 & 17.8 & & $46(42)$ & $57(54)$ \\
\hline
\end{tabular}

\subsection{Results - Fast Generators}

The effect of fast generators is tested by allowing the commitment status to change for all generators with a minimum down time less than or equal to four hours. This translates to $13 \%$ of capacity from conventional generators. Treating these generators as fast greatly improves performance for the baseline policies: modeling $W=20 \%$ yields an average regret of $1.2 \%$. This regret is half of the results without fast generators and leaves little room for improvement. Indeed, coupling capacity response sets with reserve requirements of $W=10 \%$ and $\bar{R}=5 \%$ yields an average regret of $1.3 \%$. These results corroborate [19] and [20] that the existence of fast generators can greatly improve the performance of simple deterministic models and leave little room for improvement.

The policy implication is that fast generators can greatly improve performance to the point that advanced models may offer little improvement to expected operating costs. Nonetheless, the continued existence of these approaches in CAISO and ISO-NE demonstrates the practical benefit of capacity requirements. The proposed approach can consider fast generators for generality and to provide operational continuity in changing systems (e.g., those moving towards a greater number of fast generators).

\section{Conclusion}

Power systems are facing greater uncertainty from growing penetrations of wind generation. The intermittency of wind implies that more aggressive rebalancing will be necessary to maintain system frequency. Capacity requirements are used by industry today to mitigate uncertainty, but the literature lacks ways to formally determine such capacity requirements. This work introduces capacity response 
sets and provides an automated way to determine capacity requirements based on location, ramping capability, and cost.

Testing on the IEEE 73-bus system shows the algorithm's ability to reduce scenario costs compared to other deterministic policies from the literature. The approach is particularly beneficial when dayahead commitments are fixed, providing expected cost improvements around 0.5\%. Response sets provide an efficient way to anticipate what capacity will be available without solving a stochastic model.

Future research may investigate techniques to improve convergence times. An obvious approach would be to pre-disqualify capacity ahead of time so that fewer iterations are needed. CAISO already allows for capacity disqualification in (15) and presumably has experience setting that value. There is potential for an algorithmic approach to pre-disqualify capacity. Computational performance may also improve by tweaking the pruning algorithm to generate larger, more restrictive, cuts in early iterations.

There may be opportunities to adapt response sets for different purposes. Future work may investigate disqualifying capacity to help ensure operators meet emissions targets. Future work may also focus on different types of uncertainties, such as capacity constraints to cover sudden natural gas shortages or quick reserve products to cover short-term wind uncertainty (in the range of 5-20 minutes).

\section{Acknowledgement}

The authors would like to thank the USA National Science Foundation for their support and funding of grant 1333646. The authors would also like to thank the Power Systems Engineering Research Center for their support. The anonymous reviewers also went a long way to improving the paper through their careful questions and suggestions.

\section{Nomenclature}

Indices $s$ and $t$ denote scenario and period respectively.

Sets

$G \quad$ Generators; $G(n) \subseteq G$ are located at node $n ; G^{\text {fast }} \subseteq G$ are fast generators.

L Transmission branches.

N Nodes.

T Time periods.

$S \quad$ Scenarios; $\tilde{S} \subseteq S$ are scenarios whose response sets are to be pruned during the current iteration. 


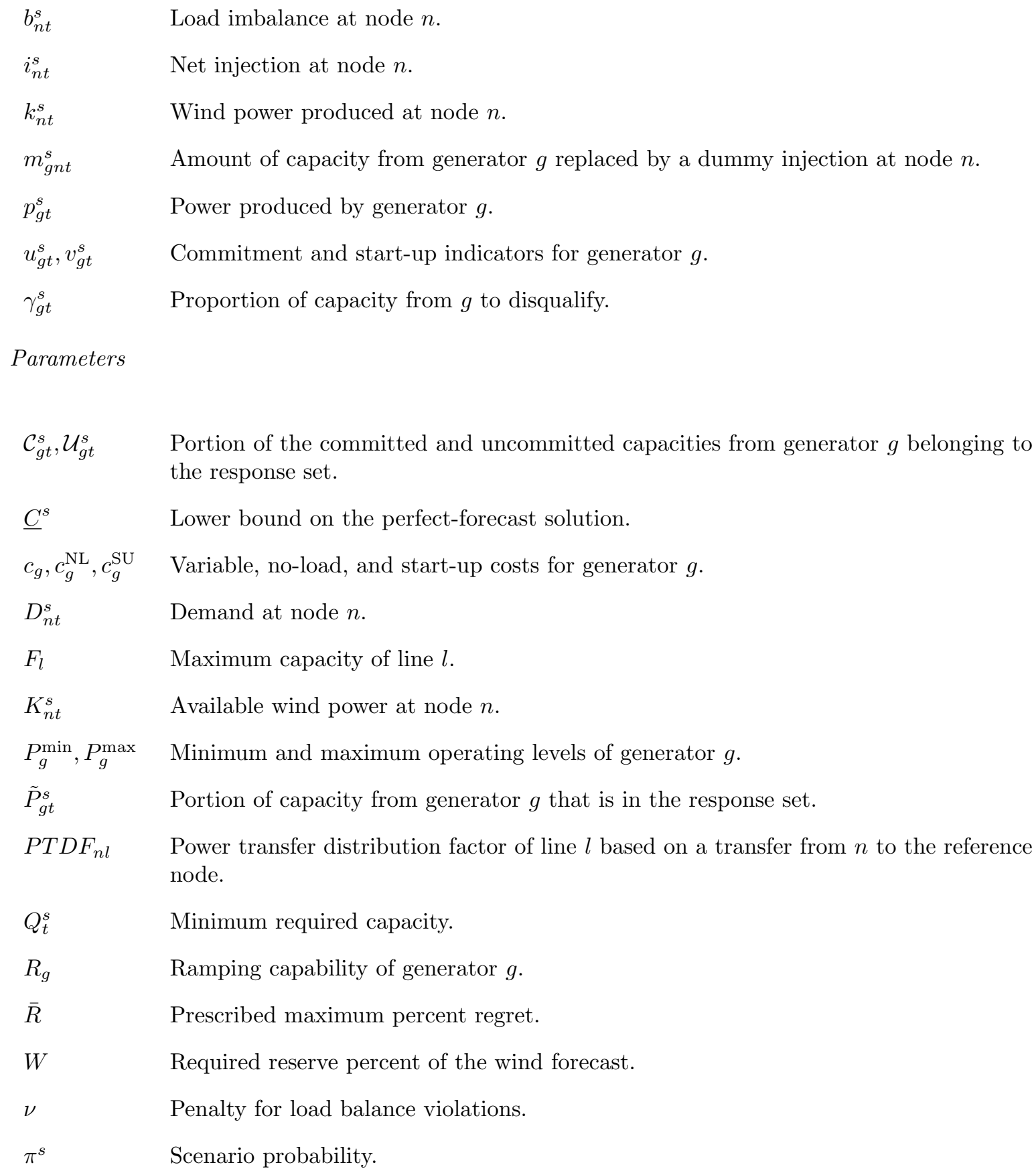

Functions

$c^{s}(\boldsymbol{x}) \quad$ Cost for scenario $s$ given decision vector $\boldsymbol{x}$.

$f(\boldsymbol{x}) \quad$ Objective function given decision vector $\boldsymbol{x}$. 


\section{References}

[1] California Public Utilities Commission, "California renewables portfolio standard (RPS)," 2013. Accessed: 2013-8-1.

[2] B.-M. Hodge, A. Florita, K. Orwig, D. Lew, and M. Milligan, "A comparison of wind power and load forecasting error distributions," tech. rep., National Renewable Energy Laboratory, 52012.

[3] L. Wu, M. Shahidehpour, and T. Li, "Stochastic security-constrained unit commitment," IEEE Trans. Power Syst., vol. 22, pp. 800-811, 52007.

[4] L. Wu, M. Shahidehpour, and T. Li, "Cost of reliability analysis based on stochastic unit commitment," IEEE Trans. Power Syst., vol. 23, pp. 1364-1374, 82008.

[5] P. A. Ruiz, C. R. Philbrick, E. Zak, K. W. Cheung, and P. W. Sauer, "Uncertainty management in the unit commitment problem," IEEE Trans. Power Syst., vol. 24, pp. 642-651, 52009.

[6] A. Papavasiliou, S. S. Oren, and R. P. O'Neill, "Reserve requirements for wind power integration: A scenario-based stochastic programming framework," IEEE Trans. Power Syst., vol. 26, pp. 21972206, 112011.

[7] J. M. Morales, A. J. Conejo, and J. Pérez-Ruiz, "Economic valuation of reserves in power systems with high penetration of wind power," IEEE Trans. Power Syst., vol. 24, pp. 900-910, 52009.

[8] A. Papavasiliou and S. S. Oren, "Multiarea stochastic unit commitment for high wind penetration in a transmission constrained network," Operations Research, vol. 61, pp. 578-592, 52013.

[9] R. Jiang, J. Wang, G. Li, and Y. Guan, "Robust unit commitment with wind power and pumped storage hydro," IEEE Trans. Power Syst., vol. 27, pp. 800-810, 52012.

[10] R. Jiang, M. Zhang, G. Li, and Y. Guan, "Two-stage network constrained robust unit commitment problem," Eur. Jour. of Oper. Res., vol. 234, 52014.

[11] D. Bertsimas, E. Litvinov, X. A. Sun, J. Zhao, and T. Zheng, "Adaptive robust optimization for the security constrained unit commitment problem," IEEE Trans. Power Syst., vol. 28, pp. 52-63, 22013.

[12] Q. Wang, X. Wang, K. Cheung, Y. Guan, and F. S. Bresler, "A two-stage robust optimization for PJM look-ahead unit commitment," in IEEE Power Tech, (Grenoble), pp. 1-6, 62013.

[13] R. Sioshansi and W. Short, "Evaluating the impacts of real-time pricing on the usage of wind generation," IEEE Trans. Power Syst., vol. 24, pp. 516-524, 52009. 
[14] R. Doherty and M. O'Malley, "A new approach to quantify reserve demand in systems with significant installed wind capacity," IEEE Trans. Power Syst., vol. 20, pp. 587-595, 52005.

[15] K. D. Vos and J. Driesen, "Dynamic operating reserve strategies for wind power integration," IEEE Trans. Power Syst., vol. 8, pp. 598-610, 82014.

[16] CAISO, "Technical bulletin: Minimum online commitment constraint," 12010.

[17] C. Parent, "Effects of minimum commitment constraints to the day-ahead and real-time markets," 122013.

[18] J. D. Lyon, M. Zhang, and K. W. Hedman, "Locational reserve disqualification for distinct scenarios," IEEE Trans. Power Syst., vol. 30, pp. 257-364, 52014.

[19] A. Tuohy, P. Meibom, E. Denny, and M. O'Malley, "Unit commitment for systems with significant wind penetration," IEEE Trans. Power Syst., vol. 24, pp. 592-601, 52009.

[20] J. Xiao, B.-M. S. Hodge, J. F. Pekny, and G. V. Reklaitis, "Operating reserve policies with high wind power penetration," Computers and Chem. Eng., vol. 35, pp. 1876-1885, 92011.

[21] L. Zhao and B. Zeng, "An exact algorithm for two-stage robust optimization with mixed integer recourse problems." Unpublished, 12012.

[22] B. Stott, J. Jardim, and O. Alsaç, "DC power flow revisited," IEEE Trans. Power Syst., vol. 24, pp. 1290-1300, 82009.

[23] M. Hedayati-Mehdiabadi, J. Zhang, and K. W. Hedman, "Wind power dispatch margin for flexible energy and reserve scheduling with increased wind generation," IEEE Trans. Sustain. Energy, vol. 6, pp. 1543-1552, 82015.

[24] A. J. Wood and B. F. Wollenberg, Power Generation, Operation, and Control. New York: John Wiley and Sons, Inc., 2 ed., 1996.

[25] M. J. Sullivan, M. Mercurio, J. Schellenberg, and Freeman, Sullivan \& Co., "Estimated value of service reliability for electric utility customers in the United States," tech. rep., Lawrence Berkeley National Laboratory, 62009.

[26] Y. Al-Abdullah, M. A. Khorsand, and K. W. Hedman, "Analyzing the impacts of out-of-market corrections," in IREP Symp., (Rethymno), pp. 1-10, 82013. 
[27] L. T. Anstine, R. Burke, J. Casey, R. Holgate, R. S. John, and H. G. Stewart, "Application of probability methods to the determination of spinning reserve requirements for the PennsylvaniaNew Jersey-Maryland Interconnection," IEEE Trans. Power App. Syst., vol. 82, pp. 726-735, 10 1963.

[28] C. Parent, "Net commitment period compensation (NCPC) LSCPR cost allocation," 112013. Market Committee Presentation.

[29] Y. Chen, X. Wang, and Q. Wang, "Overcoming computational challenges on large scale security constrained unit commitment (SCUC) problems - MISO and Alstom's experience with MIP solver," 6 2014. Presented at FERC technical conference on increasing real-time and day-ahead market efficiency through improved software.

[30] R. Jiang, J. Wang, M. Zhang, and Y. Guan, "Two-stage minimax regret robust unit commitment," IEEE Trans. Power Syst., vol. 28, pp. 2271-2282, 82013.

[31] C. Grigg et al., "The IEEE reliability test system - 1996," IEEE Trans. Power Syst., vol. 14, pp. 1010-1020, 81999 .

[32] Univ. of Washington, "Power systems test case archive," 61999.

[33] K. W. Hedman, M. C. Ferris, R. P. O'Neill, E. B. Fisher, and S. S. Oren, "Co-optimization of generation unit commitment and transmission switching with N-1 reliability," IEEE Trans. Power Syst., vol. 25, pp. 1052-1063, 52010.

[34] J. McCalley et al., "Probabilistic security assessment for power system operations," in IEEE PES General Meeting, vol. 1, pp. 212-220, 62004.

[35] A. L. Motto, F. D. Galiana, A. J. Conejo, and J. M. Arroyo, "Network-constrained multiperiod auction for a pool-based electricity market," IEEE Trans. Power Syst., vol. 17, pp. 646-653, 8 2002.

[36] J. D. Lyon, K. W. Hedman, and M. Zhang, "Reserve requirements to efficiently manage intra-zonal congestion," IEEE Trans. Power Syst., vol. 29, pp. 251-258, 12014.

[37] J. D. Lyon, F. Wang, K. W. Hedman, and M. Zhang, "Market implications and pricing of dynamic reserve policies for systems with renewables," IEEE Trans. Power Syst., vol. 30, pp. 1593-1602, 1 2015.

[38] National Renewable Energy Laboratory, "Western wind resource dataset," 2008. Accessed December, 2013. 
[39] D. Rajan and S. Takriti, "Minimum up/down polytopes of the unit commitment problem with start-up costs," tech. rep., IBM Research, 122005.

[40] H. Holttinen et al., "Methodologies to determine operating reserves due to increased wind power," IEEE Trans. Sustain. Energy, vol. 3, pp. 713-723, 102012.

[41] N. Gröwe-Kuska, H. Heitsch, and W. Römisch, "Scenario reduction and scenario tree construction for power management problems," in IEEE Power Tech, (Bologna, Italy), pp. 1-7, 62003.

[42] D. C. Montgomery and G. C. Runger, Applied Statistics and Probability for Engineers. New York: John Wiley and Sons, Inc., 5 ed., 2010.

[43] M. Tahanan, W. van Ackooij, A. Frangioni, and F. Lacalandra, "Large-scale unit commitment under uncertainty: A literature survey," tech. rep., Universitá di Pisa, 12014.

[44] Q. P. Zheng, J. Wang, and A. L. Liu, "Stochastic optimization for unit commitment - A review," IEEE Trans. Power Syst., 9 2014. , accepted for publication. 